(C) 2017 IEEE. Personal use of this material is permitted. Permission from IEEE must be obtained for all other uses, in any current or future media, including reprinting/ republishing this material for advertising or promotional purposes, creating new collective works, for resale or redistribution to servers or lists, or reuse of any copyrighted component of this work in other works.

\title{
SDN-based Traffic Engineering for Improved Resilience in Integrated Satellite-Terrestrial Backhaul Networks
}

\author{
F. Mendoza, R. Ferrús, O. Sallent \\ Universitat Politècnica de Catalunya (UPC) \\ [jesus.fabian.mendoza, ferrus, sallent]@tsc.upc.edu
}

\begin{abstract}
Resilience and high availability are considered as essential requirements in 5G networks. To fullfil these requirements, the integration of a satellite component within mobile backhaul networks arises as a compelling proposition to provide backup connectivity to critical cell sites and divert traffic from congested areas so that a limited capacity in their terrestrial links could be supplemented during peak-time or even replaced in case of total/partial failure or maintenance. This is especially of interest for public protection and disaster relief (PPDR) communications in remote/rural areas that might require the fast deployment of nework capacity as well as in distressed areas where the terrestrial backhaul infrastructure might have suffered damages. This paper first describes an architectural framework that enables the integration and management of the satellite capacity as a constituent part of a Software Defined Networking (SDN) -based traffic engineered mobile backhaul network. Then, a SDN-based Traffic Engineering (TE) application is proposed to manage some amount of dynamically steerable satellite capacity provisioned for resilience purposes to maximize a network utility function under both failure and nonfailure conditions in the terrestrial links. Numerical results are presented to assess the benefits of the proposed TE application and its performance is compared to that of a traditional overflow solution.
\end{abstract}

Keywords-Hybrid Satellite-Terrestrial Backhaul Networks; Traffic Distribution Strategies; Resilience schemes; 5G mobile networks.

\section{INTRODUCTION}

The role that satellite communications can play in the forthcoming $5 \mathrm{G}$ ecosystem is being revisited [1][2]. Ubiquitous broadband connectivity, extended to rural and lowdensity areas as well as long-haul transportation (e.g. aircraft, trains), is recognized as a key requirement for 5G [3]. Furthermore, as $5 \mathrm{G}$ networks are envisioned to increasingly be used as the primary means for delivering applications with high availability needs in many sectors such as critical infrastructures, manufacturing, emergency communications, automotive, health, etc [4][5], 5G technologies and solutions are expected to be able to achieve network availability levels in the range of five nines (i.e. $99.999 \%$ of availability) [4].

The high dependability feature attributed to satellite communications is regarded as a compelling proposition to be exploited to fulfil these requirements, increasing the availability and resiliency of mobile backhaul networks, complementing the terrestrial links that are commonly more susceptible to failures due to natural or man-made disasters [2]. This is especially of interest in public protection and disaster relief (PPDR) efforts given the high dependability on communication systems for effective disaster mitigation [6]. One of the ways to minimize the network vulnerabilities in case of a disaster is introducing an appropriate redundancy within the network [6]. In this case, satellite capacity can be deployed as a redundant backhaul capacity to any network node and also may be available to operate in challenging post disaster scenarios, allowing rapid emergency communication network deployments as those based on vehicular or transportable network nodes (referred to as mobile cells) described in [6] e.g., base stations (BS). Satellite links could provide additional bandwidth to backup connectivity to critical cell sites as well as to divert traffic from congested areas so that the capacity in the terrestrial links could be supplemented during peak-times or even replaced in case of total/partial failures as well as for emergency mobile cell deployments. Morever, it's worth noting that PPDR agencies are progressively adopting new data and video applications running on both private and public mobile broadband networks to carry out their tasks [7].

Remarkably, the roles and benefits of satellite networks in $5 \mathrm{G}$ have been introduced and discussed in 3GPP, with several use cases identified in many study items (e.g. support of "5G connectivity via satellite" within 3GPP TR23.799, the "Higher availability" requirement within 3GPP TR22.862, etc.). As a result, a requirement for $3 \mathrm{GPP}$ systems to be able to provide services using satellite access has been included within the normative Stage 1 requirements for next generation mobile telecommunications being elaborated by 3GPP [8].

In this context, the evolution of satellite ground segment systems (e.g. satellite gateways and terminals) from today's rather closed solutions towards more open architectures based on Software Defined Networking (SDN) and Network Function Virtualization (NFV) technologies arises as a necessary step [9][10], not only to bring into the satellite domain the benefits associated with the advances in network softwarization technologies that are being consolidated within the $5 \mathrm{G}$ landscape, but also to greatly facilitate the seamless 
integration and operation of combined satellite and terrestrial networks [11]. In particular, terrestrial 5G systems are widely embracing SDN technologies for enabling a unified, vendorneutral control and management of networking functions. Therefore, satellite networks shall be outfitted with a set of control and management functions and interfaces (API and/or network protocols) compatible with the mainstream SDN architectures and technologies being adopted in $5 \mathrm{G}$ in order to realize a full End-to-End (E2E) networking concept where the whole satellite-terrestrial network behavior can be programmed in a consistent and interoperable manner.

Based on the utility framework model presented in our previous work [12], this paper first describes an architectural framework that enables the integration and management of the satellite capacity as a constituent part of a SDN-based traffic engineered mobile backhaul network. Then, the paper also develops a Traffic Engineering (TE) application based in a centralized control for managing dynamically a steerable satellite capacity provisioned for resilience or emmergency purposes. Unlike more basic strategies based in a distributed control that might be devised for simply replacing a failed terrestrial link with satellite capacity, or just activating traffic overflowing through satellite in high demanding peak-times [13][14], the proposed scheme pursues an optimal allocation of the available satellite and terrestrial capacity so that a overall network utility is maximized under both failure and non-failure conditions in the terrestrial links or mobile cell deployments. The proposed SDN-based TE application is assessed and compared against a traditional overflow solution under different scenarios (different number of BSs with no terrestrial link availability).

The rest of the paper is organized as follows. Section II presents a functional view for the integration of the SDNcapable satellite network within the mobile network. Section III describes the proposed TE application. Performance results and conclusions are then presented in Section IV and V, respectively.

\section{INTEGRATION APPROACH FOR E2E TRAFFIC ENGINEERING}

Fig. 1 depicts the functional view of the integration approach for an SDN-capable satellite network, referred to as Virtualized Satellite Network (VSN) that is used within a mobile backhaul infrastructure. The integration approach is founded on two main concepts:

-Abstraction of the overall VSN as a SDN-capable "switch". In particular, the OpenFlow switch abstraction model [15] is considered to model the operation of the VSN as seen from an external controller entity. Internally, the VSN comprises the set of Satellite Terminals (ST) and gateways that are interconnected with the outside world through standard switching/routing functions used to provide L3 services such as IP/MPLS or L2 services such as Carrier Ethernet Services (CES). It is assumed that the VSN SDN controller inside the VSN orchestrates the operation of both switching/routing functions at the edges and the activation/deactivation of the satellite connections and its configuration through the VSN Network Control Centre (NCC). This SDN controller is the one that exposes an abstract view of the VSN through the OpenFlow interface.

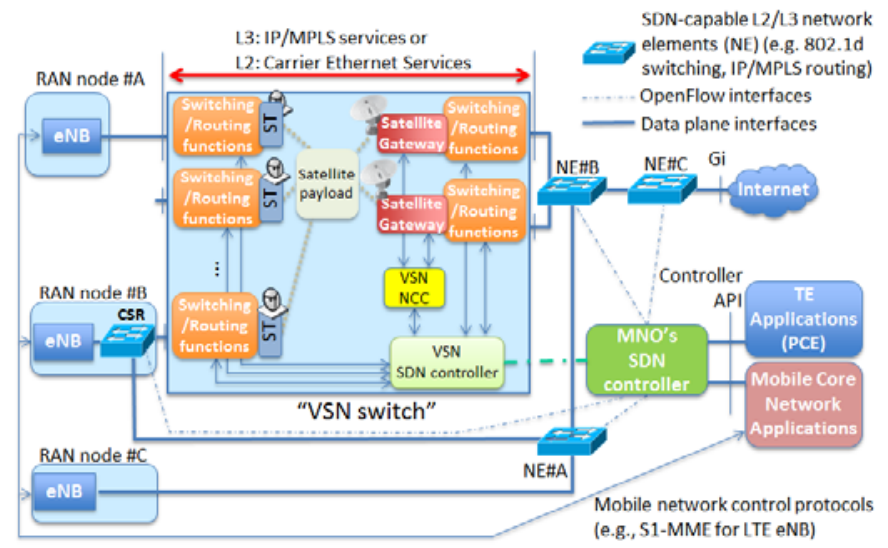

Fig. 1. Functional view and illustrative network topology considered for the development of the TE procedures.

-Use of SDN-based TE applications, with a central Path Computation Engine (PCE) that support the operation of the Mobile Core Network (MCN) for traffic management within the backhaul transport network. It is assumed that the overall transport network is managed as a single logical forwarding domain and that, inside the forwarding domain, a Mobile Network Operator (MNO)'s SDN controller makes the forwarding decisions. All SDN-capable L2/L3 Network Elements (NEs) are connected to the MNO's SDN network controller through OpenFlow interfaces, including the "VSN switch". In this way, SDN-based TE mechanisms can seamlessly span the whole network. For the terrestrial connection, no specific technology is assumed rather than considering that traffic flows can also be managed through SDN features.

Considering the network topology illustrated in Fig. 1, a message chart for the operation of a path computation mechanism for multi-path satellite-terrestrial traffic optimisation is provided in Fig. 2. The message chart shows how the failure of a path, or simply the congestion of a path that could cause QoS degradation, could be handled under the proposed integration approach. More specifically, the message chart shows how a previously established flow is re-routed in order to overcome a congestion/failure event. The steps are detailed as follows:

Step 1: The starting point considers that traffic from/to RAN node \#B and from/to RAN node\#C, called Traffic B and Traffic $C$ respectively, are both flowing through NE\#A, NE\#B and NE\#C. This could be assumed to be the optimal traffic path for a moderate traffic load scenario.

Step 2: Monitoring of the SDN forwarding elements within the domain is conducted by the MNO's SDN controller. These elements include the Cell Switch Router (CSR), "VSN switch" and NEs. TE needs granular real-time monitoring information to compute the most efficient routing decisions. Solutions such as the one described in [16] allows for an OpenFlow controller to have accurate monitoring of per-flow throughput, packet loss and delay metrics in order to aid TE. 
Step 3: An event that puts at risk the QoS of the established flows occurs. This could be, for example, a considerable traffic increase in RAN node $\# \mathrm{C}$ at certain time of the day that overloads the link among NE\#A and NE\#B, which is shared by Traffic B and Traffic C.

Step 4: The TE application detects the congestion situation. For example, the TE application could have set a high utilization threshold of 60 percent and low utilization threshold of 20 percent for the traffic load on the shared link. If this high threshold is exceeded, high utilization is observed and e.g. a part of Traffic B could be switched to pass through the VSN.

Step 5: Flow entries are installed to OpenFlow switches along the path by the MNO's SDN controller to re-route part of the traffic B through the satellite connection.

Step 6: While the path for traffic $\mathrm{C}$ remains unchanged, now part of traffic $\mathrm{B}$ is served through the $\mathrm{VSN}$, reducing congestion in the link between NE\#A and NE\#B.

\section{TE APPLICATION}

The centralized control, be it configuration or policy management or traffic engineering significantly simplifies hard-to-solve problems that benefit from centralized visibility. Equipped with global views of network resources and global control of the state of those resources, SDN controllers can make efficient resource allocation decisions in response to changing service demands, and to changing management, policy, and other inputs. In our case, the proposed TE application exploits:

- $\quad$ E2E path computation with selection of the terrestrial or satellite link for backhauling.

- Satellite capacity resource reservations to deal with BSs with no or limited terrestrial link backhaul capacity.

- Different allocation criteria depending on the traffic nature.

- Admission Control and Rate control to face overload and guarantee resources and minimum (committed) transmission rates per flow and group of flows.

- Utility maximization criteria, where the adequacy of handling specific flows over the terrestrial or satellite component, as well the effect of allocating more or less data rate, are accounted. In the end, the proposed TE application seeks to maximize the global network utility by deciding the path (satellite or terrestrial) and the allocated bit rate.

Both stream and elastic traffic is considered and utility functions are defined to describe the satisfaction level that is achieved when a particular flow is served with a certain bit rate. Further, the utility functions are defined to account for the impact on QoS due to the use of terrestrial or satellite backhaul links (i.e. the higher delay incurred when using a satellite link can result in some level of service degradation that is reflected with a lower utility). As justified in [17], the utility of stream flows, referred to as Guaranteed Bit Rate (GBR) flows in the following, is characterised by a step function, with a maximum unit utility per flow achieved at bit rate $R^{G B R}$. On the other hand, the utility of elastic flows, referred to as Non GBR flows (N-GBR), is a logarithmic function as commonly done for this type of traffic [18], with a maximum unit utility per flow achieved at bit rate $R^{N-G B R}$. In addition, it is introduced the parameters $0<p^{G B R} \leq 1$ and $0<p^{N-}$ $G B R \leq 1$ as the utility reduction factors to account for the potential quality/satisfaction degradation when using satellite links instead of terrestrial for GBR and N-GBR flows respectively.

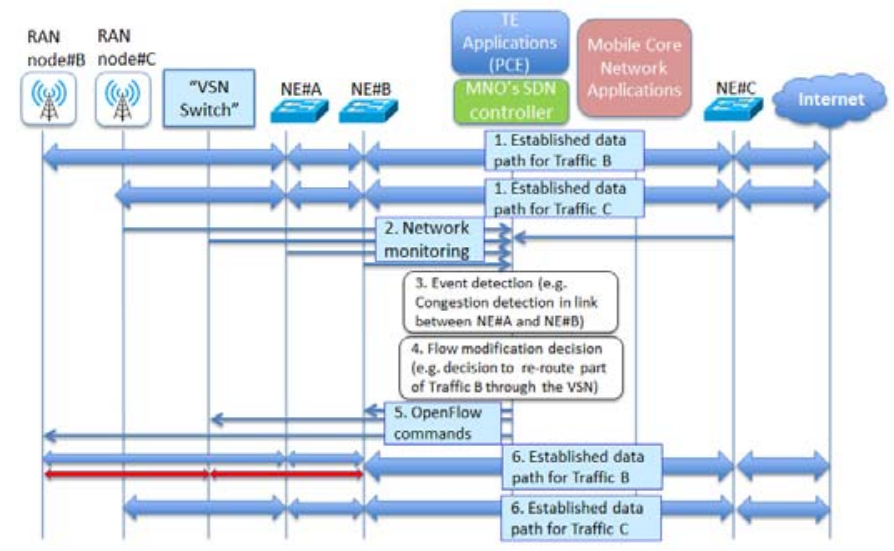

Fig. 2. TE mechanism for update an already established flow to overcome a congestion/failure event.

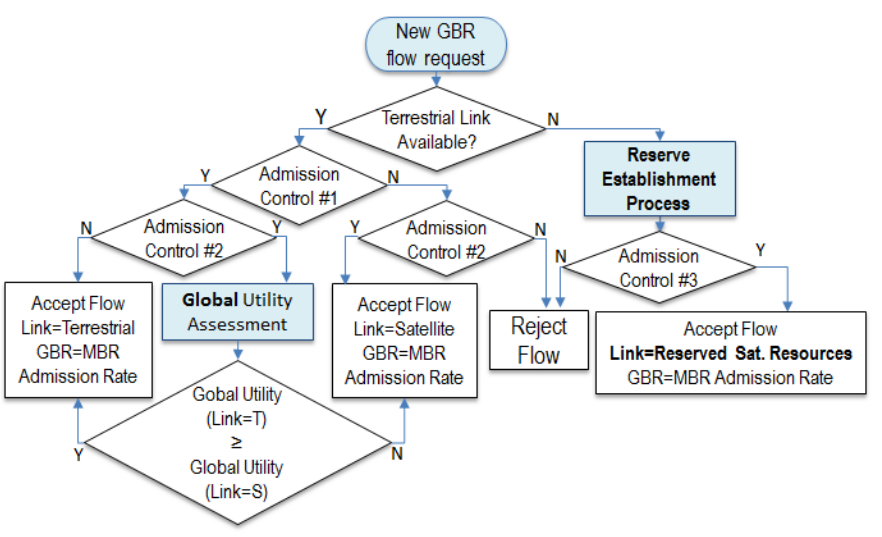

Fig. 3. TE decision-making logic for GBR traffic.

Fig. 3 shows the TE decision making logic to handle a GBR flow request. When a new GBR connection arrives at a BS with terrestrial capacity, the algorithm first goes through Admission Controls 1 and 2 ensuring that there is sufficient capacity in both terrestrial and satellite links respectively to serve the new connection without compromising available resources for the current GBR connections nor exceeding the GBR Admission Load Threshold (maximum capacity occupation of a link, allowed for use of GBR traffic). If there is sufficient backhaul capacity in both satellite and terrestrial links, the algorithm evaluates the overall utility increase achieved due to the handling the new connection by each link, choosing the link with the greatest increase in overall utility. If there is sufficient backhaul capacity in only one link (satellite or terrestrial) the new GBR connection is handled by the unique feasible option, otherwise the new GBR connection is rejected. If the new GBR connection arrives at a BS with no 
terrestrial capacity (because of e.g. a terrestrial link failure or the case of a remote/temporary BS deployment with no terrestrial capacity), a dynamic satellite resource reservation scheme is used to handle the traffic generated by that BS in order to give them some preferences in front of the use of that capacity in other BSs with both satellite and terrestrial capacity. In this case, at a new GBR connection arrival, the algorithm first checks the admission control 3, assuring there is a minimum reserved satellite capacity available to provide the service to the new connection without surpassing the NGBR admission load threshold, as well as the Maximum Reservation (maximum satellite capacity allowed for reservation purposes). The dynamic nature of the resource reservation scheme is aimed for the optimal use varying the reserved satellite capacity according to the demand at a given time. The definitions related with satellite reservation, link capacities and traffic loads as well as the admission controls calculations are presented in Tables I and II respectively.

\begin{tabular}{|c|c|c|}
\hline \multirow{2}{*}{\multicolumn{2}{|c|}{$\frac{\text { TABLE I. }}{\text { Parameter }}$}} & BASED TE APPLICATION PARA \\
\hline & & Definition \\
\hline \multicolumn{2}{|c|}{$\begin{array}{l}\text { Global GBR Satellite } \\
\text { Load }\end{array}$} & $\begin{array}{l}\text { Global satellite traffic load of GBR } \\
\text { services in BSs with terrestrial capacity. }\end{array}$ \\
\hline \multicolumn{2}{|c|}{$\begin{array}{l}\text { Global N-GBR } \\
\text { Average Flow Rate }\end{array}$} & $\begin{array}{l}\text { Average Flow Rate of all N-GBR services } \\
\text { belonging to BSs with terrestrial capacity. }\end{array}$ \\
\hline \multicolumn{2}{|c|}{$\begin{array}{l}\text { Reserved Satellite } \\
\text { Capacity (Cr) }\end{array}$} & $\begin{array}{c}\text { Satellite capacity reserved for preferential } \\
\text { use of a given BS. }\end{array}$ \\
\hline \multicolumn{2}{|c|}{$\begin{array}{l}\text { Global Reservation } \\
\text { Satellite Capacity }\end{array}$} & $\begin{array}{c}\text { Sum of Reserved Satellite Capacity of all } \\
\text { BSs with no terrestrial capacity. }\end{array}$ \\
\hline \multicolumn{2}{|c|}{$\begin{array}{l}\text { Non-Reserved } \\
\text { Satellite Capacity } \\
\text { (Cnr) }\end{array}$} & $\begin{array}{l}\text { Remaining satellite capacity after } \\
\text { reserving satellite resources, for exclusive } \\
\text { use of BSs with terrestrial capacity. }\end{array}$ \\
\hline \multicolumn{2}{|c|}{ Maximum Reservation } & $\begin{array}{l}\text { Maximum reserved satellite capacity as } \\
\text { percentage of Global Satellite Capacity }\end{array}$ \\
\hline \multicolumn{2}{|c|}{$\begin{array}{l}\text { Satellite Reservation } \\
\text { Update }\end{array}$} & $\begin{array}{l}\text { Periodicity with which the reserve values } \\
\text { are updated (sec). }\end{array}$ \\
\hline \multicolumn{2}{|c|}{$\begin{array}{l}\text { GBR Admission Load } \\
\text { Threshold }\end{array}$} & $\begin{array}{l}\text { Maximum occupation allowed for GBR } \\
\text { traffic. }\end{array}$ \\
\hline \multicolumn{2}{|c|}{ Initial Reservation } & $\begin{array}{c}\text { Minimum satellite capacity allocated as } \\
\text { reservation }\end{array}$ \\
\hline \multicolumn{3}{|c|}{ TABLE II. } \\
\hline $\begin{array}{l}\text { Admission } \\
\text { Control }\end{array}$ & & Description \\
\hline $\begin{array}{l}\text { Admission } \\
\text { Control } 1\end{array}$ & $\begin{array}{l}(\text { GBR T } \\
<(\text { GBR } \\
\text { Capacit }\end{array}$ & $\begin{array}{l}\text { rrestrial Load at BS + GBR Admission Rate) } \\
\text { Idmission Load Threshold • Terrestrial Link } \\
\text { at BS) }\end{array}$ \\
\hline $\begin{array}{l}\text { Admission } \\
\text { Control } 2\end{array}$ & $\begin{array}{l}\text { (GBR S } \\
\text { (GBR A } \\
\text { Capacit } \\
\text { AND } \\
\text { (Global } \\
<\text { (GBR }\end{array}$ & $\begin{array}{l}\text { tellite Load at BS + GBR Admission Rate) < } \\
\text { mission Load Threshold • Satellite Link } \\
\text { at BS) } \\
\text { BR Satellite Load + GBR Admission Rate) } \\
\text { dmission Load Threshold • Cnr) }\end{array}$ \\
\hline $\begin{array}{l}\text { Admission } \\
\text { Control } 3\end{array}$ & $\begin{array}{l}\text { (GBR S } \\
\text { (GBR A }\end{array}$ & $\begin{array}{l}\text { tellite Load at BS + GBR Admission Rate) }< \\
\text { mission Load Threshold } \bullet \mathrm{Cr} \text { ) }\end{array}$ \\
\hline
\end{tabular}

The TE decision making logic to handle N-GBR flow requests is depicted in Fig.4. As for GBR flows, the SDN based traffic distribution algorithm also seeks the utility maximization. To do this, at the arrival of a new N-GBR connection, the algorithm first computes the overall utility increase achieved due to the handling of the new connection by each link, choosing the link with the greatest global utility increase. Even though the link decision by connection leads to the utility maximization, the new GBR connections arrivals as well as the satellite capacity changes due to the dynamic changes of reserved satellite capacity can cause that the balance of terrestrial and satellite connections at each BS is not optimal. Due of this, the algorithm periodically (period collected by the Satellite Reservation Update parameter) calculates the optimum number of satellite and terrestrial NGBR connections per BS, matches the number of satellite and terrestrial connections at each $\mathrm{BS}$ and reallocates the right connections to the right links to maintain the optimal number of connections at each link. The dynamic reserved satellite capacity aims to ensure a minimum of resources allocated to every BS that temporarily do not have terrestrial capacity. In the presence of a BS without terrestrial capacity, the global utility can be diminished because satellite capacity can be used for BSs with terrestrial capacity, possibly leaving without satellite capacity the BSs without terrestrial capacity or distributing with no equity the resources among connections.

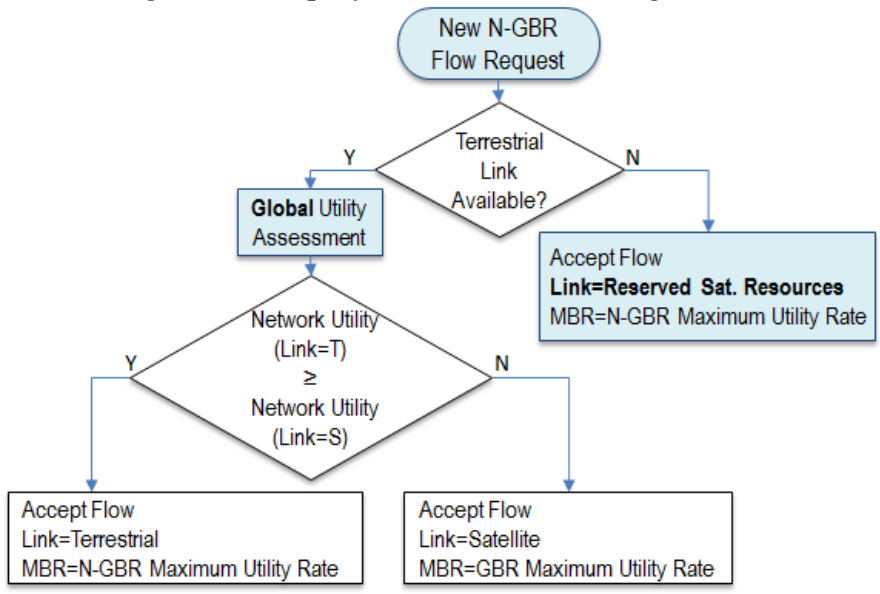

Fig. 4. TE decision-making logic for N-GBR traffic.

The scheme of Reserve Satellite Capacity establishment is shown in Fig. 5. The dynamic resource reservation mechanism is aimed to maintain as much as possible equity in the resource allocation for all GBR and N-GBR connections, regardless of whether they belong to a BS with or with no terrestrial capacity, by adjusting at discretion the reserved capacity according to changes in traffic demand, this in turn will increase the overall network utility. To do this, periodically (at time period captured in Satellite Reserve Update parameter) the reserve satellite capacity will be reestablished. The reserved satellite capacity is calculated ensuring to provide the service to the current GBR connections and ensuring that the $\mathrm{N}-\mathrm{GBR}$ connections can achieve an average bit rate equal to all others N-GBR connections in the network.

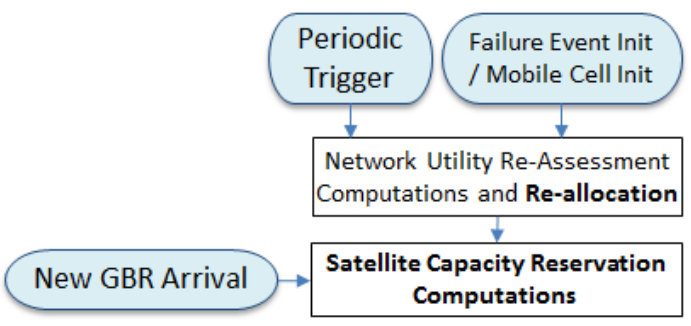

Fig. 5. TE decision-making logic for continuous monitoring and reallocation. 


\section{NUMERICAL ASSESSMENT}

\section{A. Scenario settings}

The simulation scenario considers a number of BSs with terrestrial and/or satellite backhaul capacity that serve a mix of GBR and N-GBR flows. Table III provides the range of values considered for the traffic load generation and network model parameters in the numerical assessment. With regard to the capacity of the terrestrial links, the considered setting (131 Mbps) is based on the dimensioning analysis presented in [19] to cope with the 90-th percentile of the traffic demand when considering a realistic traffic model that exhibits a log-normal distribution with an average load of $100 \mathrm{Mbps}$ per BS. This value is then considered to establish the range of values for the maximum aggregate satellite capacity $\left(\mathrm{C}_{\mathrm{S}}\right)$. On the other hand, the maximum satellite link capacity per BS is also set to 131 Mbps in line with the terrestrial capacity and considering that today's top-of-the-line satellite modems based on DVB-S2X can afford this capacity [20]. Both GBR and N-GBR traffic flows are modeled by a Poisson arrivals and exponential session duration disribution and the numerical assessment considers an execution interval of $1000 \mathrm{sec}$.

For comparison purposes, a traditional overflow strategy is considered. The strategy seeks to emulate a backhaul network with the capacity of overflow activation based only on the occupancy and bit rates measurements achieved locally (in the same BS), this is, a distributed decision making without evaluating the global network measurements (satellite and terrestrial). Table III provides the range of values considered for admission control and overflow activation/deactivation as GBR admission load threshold which determines the maximum accepted occupation of GBR traffic, the Overflow GBR Load Activation Threshold which determines the maximum terrestrial link occupation (of GBR) that activates the overflow of such kind of traffic, the Overflow N-GBR Rate Activation/Deactivation Thresholds which determines the minimum/maximum N-GBR bit rates that activates or deactivates respectively the N-GBR traffic overflow and finally the Overflow Hysteresis defined as the time after the GBR admission load threshold is exceeded to activate the GBR overflow. The network simulation as well as traffic distribution strategies are developed in matlab.

TABLE III. SCENARIO SETTINGS FOR THE NUMERICAL ASSESSMENT

\begin{tabular}{|l|c|}
\hline Network and Traffic Load Parameters & Values \\
\hline Number of BS (M) & 16 \\
\hline Number of BSs with no terr. link availability & $0-4$ \\
\hline Terrestrial link capacity at each BS (Mbps) & 131 \\
\hline Maximum Sat. link capacity per BS (Mbps) & 131 \\
\hline Maximum aggregate satellite capacity (C) as & $0,10,15,20$ \\
percentage of global terrestrial capacity. & $30 \%$ (Low), \\
\hline GBR traffic load per BS (as percentage of & $60 \%$ (Medium) \\
terrestrial deployed capacity) & $90 \%$ (High) \\
\hline & 0.218 (Low), \\
GBR flow arrival rate at each BS $(\lambda)$ & 0.436 (Medium) \\
& 0.655 (High) \\
\hline GBR mean flow duration (sec) & 30 \\
\hline N-GBR flow arrival rate at each BS $(\lambda)$ & $0.25,0.50,0.75,1.0$ \\
\hline N-GBR mean flow duration $(\mathrm{sec})$ & 20 \\
\hline
\end{tabular}

\begin{tabular}{|l|c|}
\hline High quality rate for GBR flows $\left(R^{G B R}\right)(\mathrm{Mbps})$. & $6^{*}$ \\
\hline Maximum utility N-GBR flows $\left(R^{N-G B R}\right)(\mathrm{Mbps})$. & $13^{* *}$ \\
\hline Utility Sat. reduction factor $\left(p^{G B R}\right.$ and $\left.p^{N-G B R}\right)$ & $0.6,0.8,1$ \\
\hline Overflow Strategy Parameters & 90 \\
\hline GBR admission load threshold (\%) & 80 \\
\hline Overflow GBR Load Activation Threshold (\%) & 40 \\
\hline $\begin{array}{l}\text { Overflow N-GBR Rate Activation Threshold } \\
\left.\text { (\% of } R^{N-G B R}\right)\end{array}$ & 60 \\
\hline $\begin{array}{l}\text { Overflow N-GBR Rate Deactivation Threshold } \\
\left.\text { (\% of } R^{N-G B R}\right)\end{array}$ & 5 \\
\hline Overflow Hysteresis (sec) & Values \\
\hline SDN Based Strategy Parameters & 90 \\
\hline GBR admission load threshold (\%) & 20 \\
\hline Initial Reservation (\% of C $)$ & 95 \\
\hline Maximum Reservation (\% of C s) & 1 \\
\hline Satellite Reservation Update (sec) & \\
* Typical mobile Video Resolution and Bitrates [21] & \\
** The global average for LTE download speed [22] & \\
\hline
\end{tabular}

B. Case\#1: Terrestrial and satellite backhaul capacity available in all BSs

This first assessment is intended to show the performance of the traffic distribution strategies when all terrestrial links are operational, comparing the performance of the proposed SDN based strategy and the more classical approach where the satellite capacity is used for overflow. The network performance in terms of global network utility is presented in Fig. 6. The results are presented as the global utility increase (in percentage) by both SDN based traffic distribution and overflow strategies, compared with a reference case when no satellite capacity is deployed. The results are obtained considering a medium GBR traffic load (60\%). It is observed that the increase of utility reached by the SDN based strategy is approximately $4 \%$ higher against the utility obtained by the overflow strategy, for almost any N-GBR traffic load or any maximum aggregate satellite capacity $\left(\mathrm{C}_{S}\right)$ considered. Simulations results obtained with the highest GBR traffic load (90\%), shows that the utility increase may be $10 \%$. For factors $p^{G B R}=0.6$ and $p^{N-G B R}=0.6$ (Fig. 7), the same utility gain difference among strategies is slightly higher due to the overflow strategy bases the decisions only in the parameters of overflow activation/deactivation, without considering the impact that can be presented in terms of utility in some traffic type by sending connections over satellite links, being punished by a utility decrease by a smaller factor $p$, even achieving negative utility gains respect of not having satellite capacity.

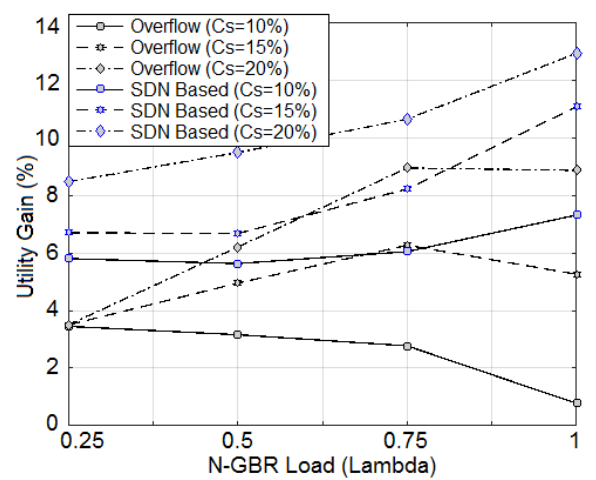

Fig. 6. Global utility gain, with a medium GBR Load and $p^{G B R}=p^{N-G B R}=1$. 


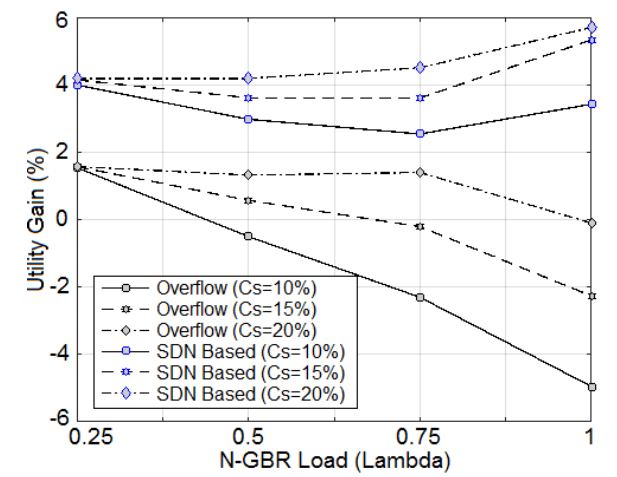

Fig. 7. Global utility gain, with a medium GBR Load and $p^{G B R}=p^{N-G B R}=0.6$.

As explained in section III, in addition to choosing the backhaul link that allows a greater increase of utility for each new connection arrival, the SDN based strategy also executes periodially the N-GBR link reallocations. These reallocations per connection during flow duration time are in the range of 0.15-0.8, depending on GBR and N-GBR traffic load and showing a tendency to decrease as N-GBR traffic increases.

The strategy performance in terms of GBR average rejection rate is showed in Fig. 8, showing significant improvements by the SDN based strategy for any N-GBR traffic load or any maximum aggregate satellite capacity.

In terms of N-GBR bit rates the results are similar under medium and high N-GBR traffic load as shown in Fig. 9. This is due to the fact that under these traffic loads almost all backhaul capacity (satellite and terrestrial) is used under any traffic distribution strategy, however, the difference lies on how this capacity is distributed among all N-GBR connections in the network, for example, under the overflow strategy we observe a substantial increase on the N-GBR bit rates standard deviation which is about in $2.8 \mathrm{Mbps}$ considering the higher N-GBR traffic load, whereas with the SDN based strategy the same measure can be only about $1 \mathrm{Mbps}$. This is due to the fact that the strategy distributes the traffic based on the global occupation measurement of both satellite and terrestrial links, as well as the connections number present in each link.

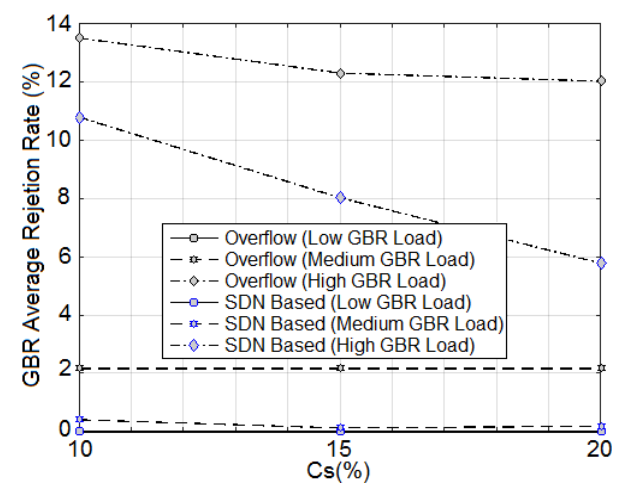

Fig. 8. GBR Average Rejection rate for $p^{G B R}=p^{N-G B R}=1$.

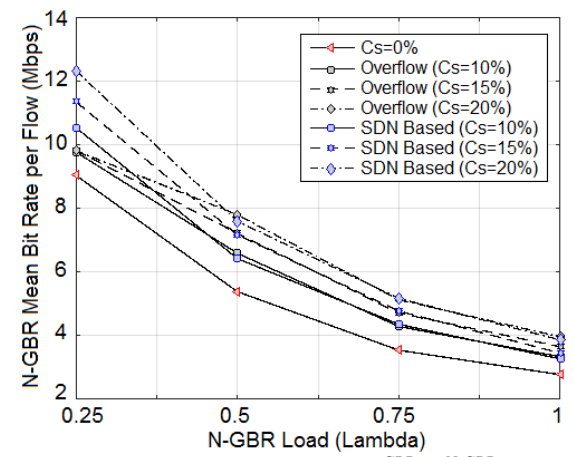

Fig. 9. Average bit rate per N-GBR flow for $p^{G B R}=p^{N-G B R}=1$.

\section{Case\#2: Terrestrial backhaul capacity not available in some BSs}

This second assessment is intended to show the performance of the traffic distribution strategies when not all terrestrial links are operational, especially in BSs that temporarily face a lack of terrestrial link availability.

First, we assess the network considering one BS without terrestrial capacity, fixing the traffic load at medium GBR traffic Load (60\%), the highest N-GBR traffic load $(\lambda=1)$ and $\mathrm{Cs}=20 \%$. The results are compared to those obtained when evaluating a network under the same load and where all BSs have terrestrial capacity available. Fig. 10-12 show the impact in terms of utility, GBR rejection rate and N-GBR bit rates, respectively, when one $\mathrm{BS}$ without terrestrial capacity is present in the network. The performance is showed for BSs where there is no terrestrial capacity (BS with no TC), in BSs where there is terrestrial capacity (BSs with TC) and results are compared with the performance reached in a network where there is terrestrial link availability in all BSs (full terrestrial availability). Each measure is compared to the case where there is no available satellite capacity $\left(\mathrm{C}_{S}=0\right)$.

Although the results obtained in BSs with terrestrial capacity do not show a significant impact respect to the case of a network with a full terrestrial availability, it is not so for the BS that temporarily presents a terrestrial failure. We see in Fig. 10, that the utility values in the BS with terrestrial failure are reduced by about $20 \%$ under the conventional overflow strategy, while the SDN based strategy is able to keep the same utility levels reached in a network that does not present any terrestrial link failure. The only drawback is the number of mean N-GBR reallocations per connection that is 0.59 .

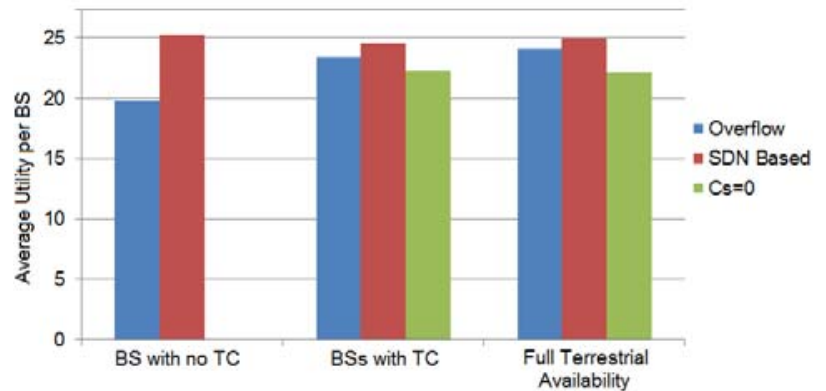

Fig. 10. Average utility for GBR medium traffic load, N-GBR flow arrival rate $\lambda=1$ and $p^{G B R}=1$ and $p^{N-G B R}=1$. 


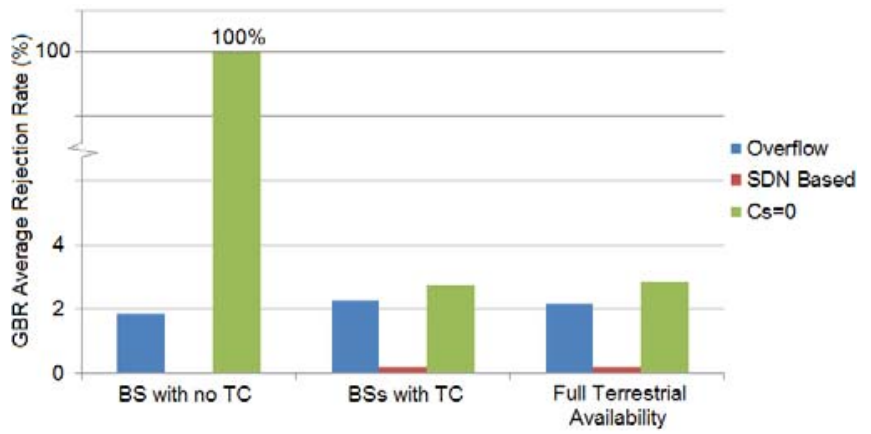

Fig. 11. GBR average rejection rate for GBR medium traffic load, N-GBR flow arrival rate $\lambda=1$ and $p^{G B R}=1$ and $p^{N-G B R}=1$.

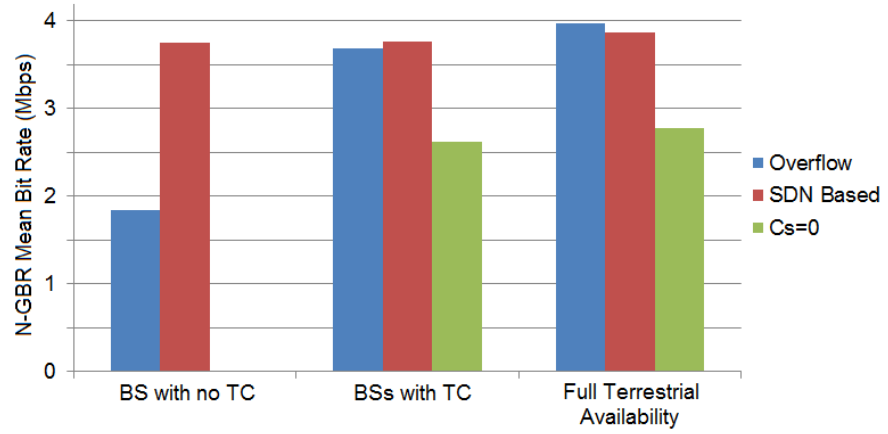

Fig. 12. N-GBR mean bit rate for GBR medium traffic load, N-GBR flow arrival rate $\lambda=1$ and $p^{G B R}=1$ and $p^{N-G B R}=1$.

The results in terms of GBR rejection rate (Fig. 11) also show how through the application of the SDN strategy the rejection rate is mitigated in the $\mathrm{BS}$ with no terrestrial capacity, while under the application of the overflow strategy the rejection rate only decreases slightly.

The most noticeable difference is observed in the N-GBR mean bit rate (Fig 12), where under the application of the overflow strategy the N-GBR mean bit rate is diminished by more than half that obtained by the SDN strategy. This value becomes more relevant if we take into account that under the SDN based strategy there are also no GBR rejections.

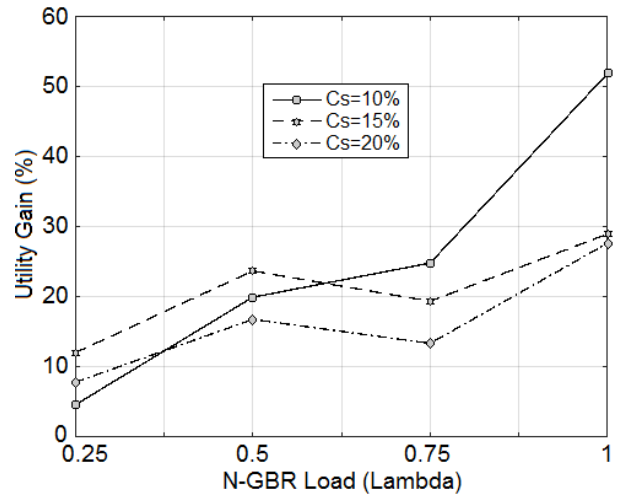

Fig. 13. SDN based strategy utility gain over Overflow strategy at the BS with no terrestrial availability for GBR medium traffic load and $p^{G B R}=1$ and $p^{N-}$ $G B R=1$.

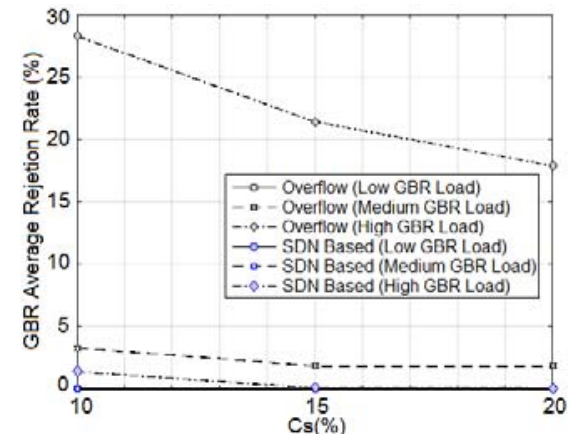

Fig. 14. GBR average rejection rate at the BS with no terrestrial link $\left(p^{G B R}=1\right.$ and $\left.p^{N-G B R}=1\right)$.

Focus on BSs with no terrestrial capacity, we assess the network under 4 different N-GBR traffic loads, 3 different satellite system capacities $(\mathrm{Cs}=10 \%, 15 \%, 20 \%)$, and we fix the GBR load at $60 \%$. Fig. 13 shows the utility performance of the strategies in the BS with no terrestrial capacity. The figure shows directly the utility gain in percentage obtained by the SDN strategy respect to the achieved by the overflow strategy, reaching a utility gain of up to $50 \%$ with a medium GBR traffic load or even in simulations for a high GBR traffic load, this utility can reach up to $85 \%$.

The GBR average rejection rate is showed in Fig. 14, showing a significant rejection rate decrease by the SDN based strategy. We observe that in spite of accepting a larger number of GBR connections, the SDN based strategy still achieves a N-GBR utility gain of up to $150 \%$ for a medium GBR load respect the achieved by the overflow strategy (Fig. 15). The utility gains can be even greater up to $350 \%$ for a high GBR load. SDN based strategy has the ability to distribute all connections in such a way that a similar rate is reached among them, allowing through the reserved resources for BSs that temporarily face a lack of terrestrial capacity, a resource allocation fairness among all N-GBR, regardless of their BS terrestrial link availability.

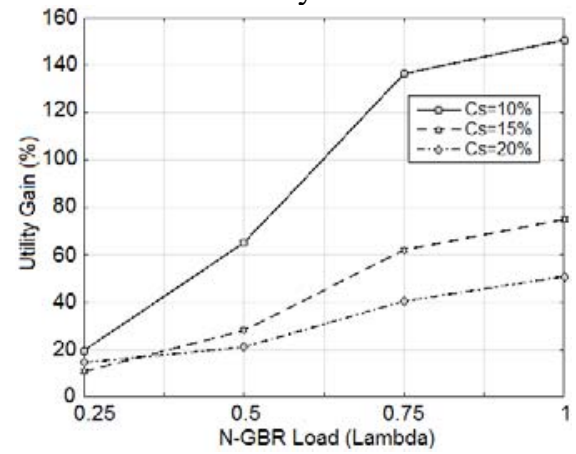

Fig. 15. N-GBR Average Utility increase under failure conditions at BS with no terrestrial capacity $\left(p^{G B R}=1\right.$ and $\left.p^{N-G B R}=1\right)$.

Finally, let us consider the case that the same amount of satellite capacity provisioned to be shared in normal conditions among $16 \mathrm{BSs}$ with terrestrial backhaul is used to serve a higher number of BS with no terrestrial link availability. This could be a situation where a number of transportable BSs with only satellite backhaul are brought into an incident area or the case that a disaster has severely impaired the terrestrial backhaul infrastructure of multiple 
BSs. Fig. 16 provides results for up to 4 BSs with no terrestrial capacity in a group of 16 BSs. Medium GBR load, N-GBR load with $\lambda_{\mathrm{UN}}=1$ flows $/ \mathrm{s}$ and $\mathrm{Cs}=20 \%$ are considered. As expected, global network utility decreases with a higher number of BSs with no terrestrial link availability. However, it could be seen that the SDN-based strategy is able to deliver higher utilities than the overflow strategy. Similar results are observed in terms of N-GBR mean bit rates, achieving a range of 5.1-3.2 Mbps for 0 to $4 \mathrm{BSs}$ with no terrestrial capacity under the Overflow strategy and of 5.3-4.2 Mbps under the SDN-based solution.

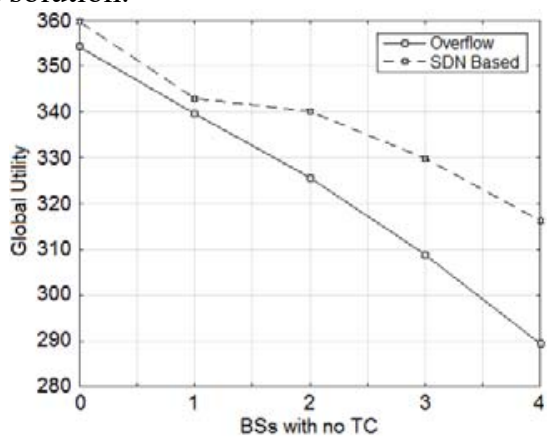

Fig. 16. Global utility for different number of BSs with no terrestrial availability $\left(p^{G B R}=p^{N-G B R}=0.8\right)$

\section{CONCLUSIONS}

The adoption of SDN and NFV technologies into the satellite domain is seen as a key facilitator to enhance the delivery of satellite communications services and achieve a better integration of the satellite component within the $5 \mathrm{G}$ ecosystem.

This paper has first outlined an integration approach for the realization of E2E TE in a mobile network where a SDNcapable satellite network forms part of the backhaul infrastructure. Under this framework, a SDN-based TE application has been developed. Thanks to centralised control, the TE application allows managing the use of the satellite capacity provisioned for resilience purposes among a number of BSs so that the overall network utility is maximized under both failure and non-failure conditions in the terrestrial links. The performance of the proposed TE application has been assessed by means of numerical simulation. Obtained results shows how overall network performance is improved, compared to that of a traditional overflow solution, in terms of network utility increase, GBR rejection rate decrease, N-GBR utility increase, and improved N-GBR fairness, especially for BSs that temporary face a lack of terrestrial link availability. Under failure conditions, it's been shown that the reservation scheme implemented within the TE application allows keeping fair utility levels in the BSs affected by terrestrial link failures.

\section{ACKNOWLEDGMENT}

Research leading to these results has received funding from the European Union's H2020 Research and Innovation Programme (H2020-ICT-2014-1) under the Grant Agreement H2020-ICT-644843 and by the Spanish Research Council and FEDER funds under RAMSES grant (ref. TEC2013-41698R).

\section{REFERENCES}

[1] NetWorld2020's " SatCom WG The role of satellites in 5G", Version 5, 31th July 2014. https://www.networld2020.eu/wpcontent/uploads/2014/02/SatCom-in-5G_v5.pdf

[2] Sacchi, C.; Bhasin, K.; Kadowaki, N.; Vong, F., "Toward the "space 2.0" Era [Guest Editorial]," Communications Magazine, IEEE , vol.53, no.3, pp.16,17, March 2015.

[3] 5G Vision - The 5G Infrastructure Public Private Partnership: the next generation of communication networks and services. https://5gppp.eu/wp-content/uploads/2015/02/5G-Vision-Brochure-v1.pdf

[4] NGMN Alliance, "5G White Paper", February 2015. https://www.ngmn.org/uploads/media/NGMN_5G_White_Paper_V1_0. pdf

[5] Osseiran A. et al., "Scenarios for 5G Mobile and Wireless Communications: The Vision of the METIS Project", IEEE Communications Magazine, May 2014.

[6] T. Gomes et al., "A survey of strategies for communication networks to protect against large-scale natural disasters", 8th International Workshop on Resilient Networks Design and Modeling (RNDM), Halmstad, Sweden, 13-15 Sept. 2016.

[7] R. Ferrús and O. Sallent, "Mobile Broadband Communications for Public Safety: The Road Ahead Through LTE Technology", Wiley and Sons, ISBN: 978-1-118-83125-0, October 2015

[8] 3GPP TS 22.261 V0.1.1 (2016-08), "Service requirements for next generation new services and markets; Stage 1 (Release 15)", August 2016.

[9] H2020 VITAL research project website at http://www.ict-vital.eu/

[10] Bertaux L. et al. , "Software Defined Networking and Virtualization for Broadband Satellite Networks". IEEE Communications Magazine, March 2015.

[11] R. Ferrus, H. Koumaras, O. Sallent, G. Agapiou, T. Rasheed, M.-A. Kourtis, C. Boustie, P. Gelard, T. Ahmed, "SDN/NFV-enabled satellite communications networks: Opportunities, scenarios and challenges", Elsevier, Physical Communication, Volume 18, Part 2, Pages 95-112. March 2016.

[12] R. Ferrus, O. Sallent, T. Ahmed, R. Fedrizzi, "Towards SDN/NFVenabled satellite ground segment systems: End-to-End Traffic Engineering Use Case", 2017 IEEE International Conference on Communications Workshops (ICC Workshops), Paris, 21-25 May 2017.

[13] C. A. Grazia et al., "Integration between terrestrial and satellite networks: the PPDR-TC vision," 2014 IEEE 10th International Conference on Wireless and Mobile Computing, Networking and Communications (WiMob), Larnaca, 2014.

[14] E. H. Fazli, M. Werner, N. Courville, M. Berioli and V. Boussemart, "Integrated GSM/WiFi Backhauling over Satellite: Flexible Solution for Emergency Communications", Vehicular Technology Conference, 2008. VTC Spring 2008. IEEE, Singapore, 2008.

[15] Open Networking Foundation, "OpenFlow Switch Specification Version 1.5.1 ( Protocol version 0x06 )", ONF TS-025, March 2015.

[16] N. L. M. van Adrichem, C. Doerr and F. A. Kuipers, "OpenNetMon: Network monitoring in OpenFlow Software-Defined Networks," 2014 IEEE Network Operations and Management Symposium (NOMS), Krakow, 2014, pp. 1-8. doi: 10.1109/NOMS.2014.6838228.

[17] F. Mendoza, R. Ferrús, O. Sallent, "A traffic distribution scheme for $5 \mathrm{G}$ resilient backhauling using integrated satellite networks", IEEE, 13th International Wireless Communications and Mobile Computing Conference, Valencia, Spain, 26-30 June 2017.

[18] E. Kelly, "Charging and rate control for elastic traffic", European Trans.Telecomm, vo1.8, pp.33-37, 1997.

[19] Mendoza F., Ferrús R., Sallent O., "Flexible Capacity and Traffic Management for Hybrid Satellite-Terrestrial Mobile Backhauling Networks", International Symposium on Wireless Communication Systems (ISWCS), Poznan, Poland, 20-23 Sept. 2016.

[20] ESA news: https //artes.esa.int/news/newtec-introduces-industrysfirst-dvb-s2x-vsat -modem. Last access on 18 November 2016.

[21] "Video as a Basic Service of LTE Networks: Mobile vMOS Defining Network Requirements". http://www.huawei.com/minisite/4-5g/en/industryjsdc-j.htm

[22] OpenSignal. "The State of LTE" February 2016. http://opensignal.com/reports/2016/02/state-of-lte-q4-2015/ 